\title{
A Mathematical Model to Expedite Electroporation Based Vaccine Development for COVID-19
}

\author{
Akshil Kumar Sharma ${ }^{1}$, Debarshi Ghosh ${ }^{2}$ (D), Nitin Kumar Saluja ${ }^{3}$, Thakur Gurjeet Singh ${ }^{4, *}$ (D) \\ 1 Chitkara University Institute of Engineering \& Technology, Chitkara University Punjab, India; akshil.sharma@gmail.com \\ (A.K.S.); \\ 2 Chitkara University Institute of Engineering \& Technology, Chitkara University Punjab, India; \\ debarshi.ghosh@chitkara.edu.in (D.G.); \\ 3 Chitkara University Institute of Engineering \& Technology, Chitkara University Punjab, India; \\ nitin.saluja@chitkara.edu.in (N.K.S.); \\ 4 Chitkara College of Pharmacy, Chitkara University Punjab, India; gurjeet.singh@ @ chitkara.edu.in (T.G.S.); \\ * Correspondence: gurjeet.singh@ chitkara.edu.in, gurjeetthakur@gmail.com (T.G.S.);
}

Received: 13.03.2021; Revised: 10.05.2021; Accepted: 12.05.2021; Published: 10.06.2021

\begin{abstract}
Electroporation has an application in the selective delivery of drugs explicitly into cells. However, the challenge is to achieve efficiency in delivering the drugs. The key parameter responsible for successful electroporation-mediated drug delivery is induced transmembrane voltage (ITMV). The Food \& Drug Administration (FDA) has recently approved the clinical trials of DNA plasmid delivery of the COVID-19 vaccine through electroporation. The requirement is to develop a COVID-19 vaccine within a limited time. Hence, the extensive amount of laboratory experiments are not feasible. It has increased dependency on simulation-based analysis. The simulations of electroporation depend on ITMV expression for the specified cell and the environment. In this paper, we have derived the closedform expression of ITMV $\left(\Delta \mathrm{V}_{\mathrm{m}}\right)$. The closed-form expression is used in COMSOL Multiphysics simulation to obtain extracellular concentration variation as a function of time. The simulation results match the empirical results from the literature and hence validate the closed-form expression. The closed-form expression will reduce the development time of electroporation-assisted COVID-19 vaccine delivery.
\end{abstract}

Keywords: electroporation; transmembrane voltage; DNA plasmid; molecular transport; drug delivery.

(C) 2021 by the authors. This article is an open-access article distributed under the terms and conditions of the Creative Commons Attribution (CC BY) license (https://creativecommons.org/licenses/by/4.0/).

\section{Introduction}

The interaction of electrical signals with a living organism depends on the different structures of the organism. A cell consists of cytoplasm, which includes the nucleus, mitochondria, and other cell organelles necessary for a life surrounded by a thin insulating layer called the plasma membrane to protect the cytoplasm extracellular stress. The equivalent electrical model of a spherical cell is regarded as an electrolyte (cytoplasm) enclosed by a dielectric shell (Plasma membrane) and is regarded as a resistive capacitance (RC) circuit. The plasma membrane is a phospholipid bilayer that controls the exchange of materials between the cytoplasm and extracellular medium [1]. The cytoplasm is an electrolyte of resistance $R_{e}$, and the plasma membrane is a spherical capacitor of capacitance $C_{m}$. The membrane consists of a lipid bilayer that controls the inflow of materials from the extracellular medium to the intracellular medium and vice-versa. The lipid bilayer further consists of ion pumps, voltage- 
gated channels, and ion transporters that are non-permeable to large molecules. The difference in ion concentration between extracellular space and intracellular space of the cell creates a potential difference (voltage) across the membrane, called trans-membrane resting voltage $\left(V_{r}\right)$. Channels or pathways in the membrane are needed to allow the entry of large molecules like DNA into the cell. Several studies have been carried out to create pathways in the membrane under the effect of electricity.

The impact of applied electric field on the structural changes in the plasma membrane to study the exchange of materials between the extracellular medium and the cytoplasm of biological cells has been carried out extensively [2,3].

It has been observed that the structural change of cell membranes exposed to the external field makes the cell-permeable and conductive. The phenomenon is called Electroporation or Electro permeabilization and has a wide range of applications such as transport of drugs, DNA molecules into the cell, insertion of proteins into the cell membrane, fusion of cells, food preservation etc. [4-8].

Application of electroporation in gene vaccine or delivery of plasmids started way back in 1982 when Neumann et al. [9] first delivered DNA using electroporation. After that, there have been several recent developments in the use of electroporation as a potent technique for delivering vaccines or other medications using electroporation [10-18]. As reported by Miklavcic et al., 2019 [19] that there are about 80 reported clinical trials in which electroporation is used for delivery of DNA plasmid or other medicines. Recently in the COVID-19 pandemic US Food \& Drug Administration (FDA) has approved clinical trials of INO 4800, a DNA plasmid vaccine with electroporation [20]. Thus, it is evident that electroporation is an effective alternative for DNA vaccine delivery, and therefore it requires a thorough understanding of the electroporation mechanism and the mathematics behind it.

When a cell is exposed to a pulsed electric field, the cell membrane is polarized [21], and induced transmembrane voltage $\left(\mathrm{V}_{\mathrm{m}}\right)$ is developed across the membrane, which superimposes on the trans-membrane resting voltage [22]. Electroporation occurs when induced trans-membrane voltage exceeds the critical threshold value $(\approx 1 \mathrm{~V})[23,24]$. The increased induced transmembrane voltage $\left(\mathrm{V}_{\mathrm{m}}\right)$ is limited by the pores created in the cell membrane.

Schwan [25] developed a theory to calculate the value of induced transmembrane voltage by considering a spherical cell exposed to a steady electric field by solving Laplace's equation. The cell's transient behavior can be described by accounting for the dielectric permittivity of the membrane $\left(\varepsilon_{\mathrm{m}}\right)$ in addition to its electrical conductivity.

In most studies, the detailed derivation of ITMV, which is the basic principle behind the study of electroporation, is not available. Therefore, this paper has presented a detailed derivation of ITMV for a single spherical cell under the electric field. Here detailed analysis of Schwann et al. [25] linear electric model with an equivalent circuit is studied in the paper with derivations that would help the community understand ITMV better. The paper further discusses a COMSOL Multiphysics model of Schwan cell to demonstrate electroporation mediated molecular transport. 


\section{Materials and Methods}

\subsection{Schwann electrical model of the biological cell.}

Foster and Schwann proposed an electrical model of a biological cell in 1998 (also known as the Schwann model [25]). According to this model, the cell consists of three regions: homogenous conducting cytoplasm (i) having tens of micrometer in diameter, a thin insulating plasma membrane (m), and extracellular medium (e), as shown in Figure 1.

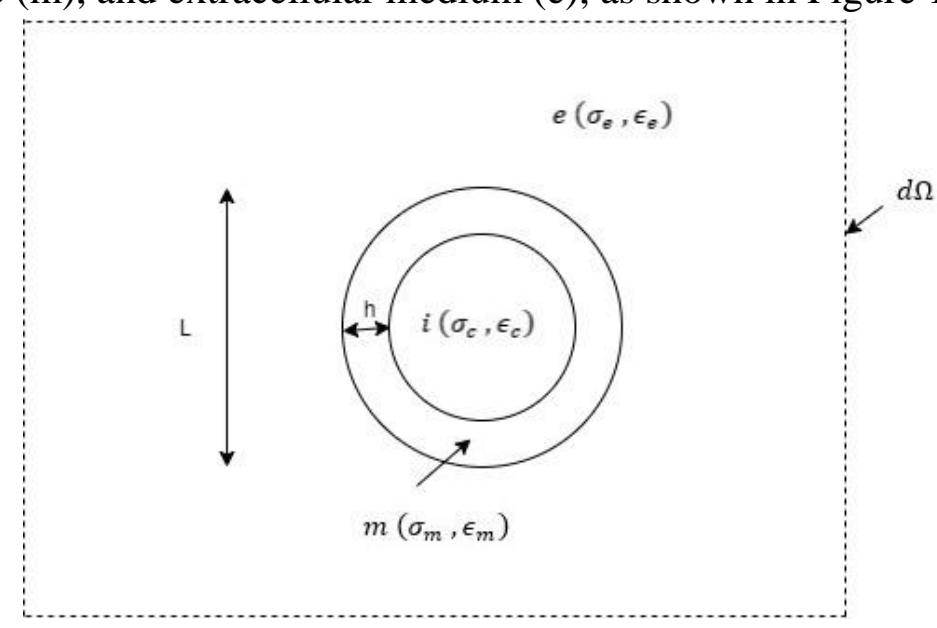

Figure 1. Schwan electrical model of a biological cell.

The typical characteristics of the three regions of the cell are given in Table 1.

Table 1. Characteristics values of a Schwann cell.

\begin{tabular}{l|l} 
Parameter & Value \\
\hline $\mathrm{L}$ & $20 \mu m$ \\
\hline $\mathrm{h}$ & $\sim 5 n m$ \\
\hline$\sigma_{c}$ & $\sim 1 S / m$ \\
\hline$\sigma_{e}$ & $\sim 1 S / m$ \\
\hline$\epsilon_{0}$ & $8.854 \times 10^{-12} C^{2} N^{-1} m^{-2}$ \\
\hline$\epsilon_{e}$ & $80 \varepsilon_{0}$ \\
\hline$\varepsilon_{c}$ & $80 \varepsilon_{0}$ \\
\hline$\varepsilon_{m}$ & $80 \varepsilon_{0}$ \\
\hline$\sigma_{m}$ & $10^{-5}-10^{-7} \mathrm{~m}$
\end{tabular}

\subsection{Electrical equivalent circuit of Schwann cell-OD model.}

In the electrical equivalent circuit, a cytoplasm is considered as an electrolyte of resistance 'Rc', plasma membrane as a spherical capacitor of capacitance ' $\mathrm{Cm}$ ', and the extracellular medium (ambient medium) as an electrolyte of resistance 'Re'. The equivalent electric circuit of a Schwann cell [19] is as shown in Figure 2.

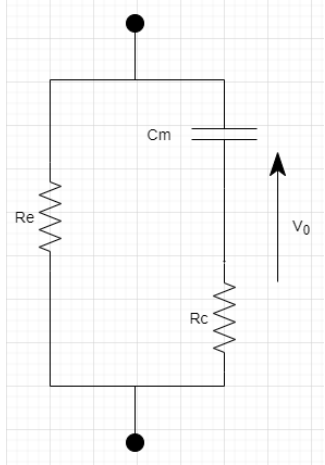

Figure 2. Equivalent circuit of Schwann cell. 
When a voltage source V0 is connected across a cell, the cell membrane's charging takes place, leading to ITMV (Vm).

Charging of cell membrane after time $t$ is given by -

$Q=Q_{0}\left(1-e^{\frac{-t}{R_{c} C_{m}}}\right)=Q_{0}\left(1-e^{\frac{-t}{\tau_{m}}}\right)$

where $\tau_{m}=R_{c}$. $C_{m}$ is the capacitive time constant.

Since $V=\frac{Q}{C}$

Therefore, $V_{m}(t)=V_{0}\left(1-e^{\frac{-t}{\tau_{m}}}\right)$

If $\mathrm{a}$ is the radius of the cell and $\mathrm{E}$ is the electric field corresponding to the voltage source Vo, then $\mathrm{E}=\frac{\mathrm{V}_{0}}{2 \mathrm{a}}$ or $\mathrm{V}_{0}=2 \mathrm{aE}$.

$$
\text { Now, } V_{m}(t)=2 a E\left(1-e^{\frac{-t}{\tau_{m}}}\right)
$$

Thus, induced transmembrane voltage increases exponentially with time. Thus, the above model depicts an intrinsic dependency of ITMV on the radius 'a' of the cell, further validated in the 3D model. The above model does not consider the effects of the cell's shape and the duration of the applied electric field. Thus, a more heuristic approach of the partial differential equation- Maxwell equations is needed to study the electric field's effect as a whole in the cell.

\section{Results and Discussion}

\subsection{Analytical derivation of induced transmembrane voltage (ITMV).}

ITMV is defined as the difference between the electric potentials on both the side of the plasma membrane.

The spatial distribution of the electric field, $\vec{E}$, is related to the distribution of space charge by Gauss's law in electrostatics.

$$
\vec{\nabla} \cdot \vec{E}=\frac{\rho}{\epsilon}
$$

where $\rho$ is the volume charge density and $\epsilon$ is the permittivity of the medium.

Since, $E=-\vec{\nabla} \cdot V$, where $\mathrm{V}$ is the electric potential so,

$\nabla^{2} \cdot V=-\frac{\rho}{\varepsilon_{0}}$

Since extracellular space and intracellular space of the cell are charge-free, $\rho=0$, So the equation (4) can be modified as,

$\nabla^{2} V=0$

Equation (5) is known as the Laplace equation, whose solution gives the steady-state spatial distribution of $\mathrm{V}$ in a given coordinate system in the cell.

In cartesian coordinate system $(\mathrm{x}, \mathrm{y}, \mathrm{z})$, Laplace equation takes the form:

$\left(\frac{\partial^{2}}{\partial x^{2}}+\frac{\partial^{2}}{\partial y^{2}}+\frac{\partial^{2}}{\partial z^{2}}\right) V(x, y, z)=0$

Or $\quad \frac{\partial^{2} V(x, y, z)}{\partial x^{2}}+\frac{\partial^{2} V(x, y, z)}{\partial y^{2}}+\frac{\partial^{2} V(x, y, z)}{\partial z^{2}}=0$

Since the cell is assumed to be spherical, so rewriting equation (6) in the spherical coordinate system as

$\frac{1}{r^{2}} \frac{\partial}{\partial r}\left(r^{2} \frac{\partial V(r, \theta, \varphi)}{\partial r}\right)+\frac{1}{r^{2} \sin \theta} \frac{\partial}{\partial \theta} \sin \theta \frac{\partial V(r, \theta, \varphi)}{\partial \theta}+\frac{1}{r^{2} \operatorname{Sin}^{2} \theta} \frac{\partial^{2} V(r, \theta, \varphi)}{\partial \varphi^{2}}=0$ 
Spherical polar coordinates are shown in Figure 3. Equation (7) is used to determine the spatial distribution of electric potential in and around the spherical cell subjected to a uniform electric field.

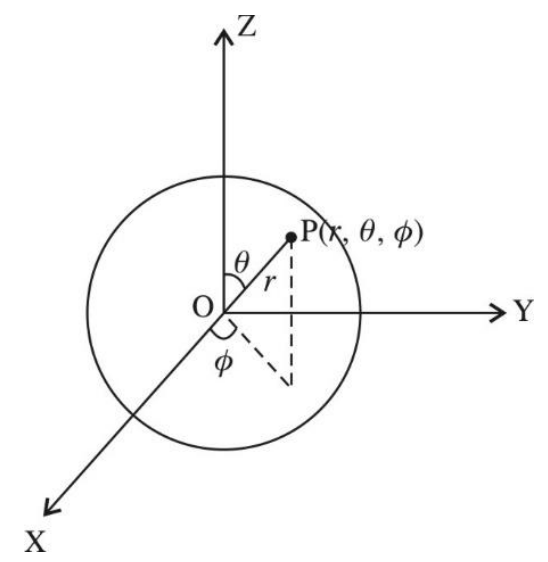

Figure 3. Spherical polar coordinate.

In the case of azimuthal symmetry, the potential $\mathrm{V}$ depends only on $\mathrm{r}$ and $\theta$. Therefore, $\frac{\partial V}{\partial \varphi}=0$.

Hence, $V(r, \theta, \varphi)$ is considered as $V(r, \theta)$ in equation (7).

So, $\frac{1}{r^{2}} \frac{\partial}{\partial r}\left(r^{2} \frac{\partial V(r, \theta)}{\partial r}+\frac{1}{r^{2} \sin \theta} \frac{\partial}{\partial \theta} \sin \theta \frac{\partial V(r, \theta)}{\partial \theta}\right)=0$

Or, $\frac{\partial^{2} V(r, \theta)}{\partial r^{2}}+\frac{2}{r} \frac{\partial V(r, \theta)}{\partial r}+\frac{1}{r^{2}} \frac{\partial^{2} V(r, \theta)}{\partial \theta^{2}}+\frac{\cot \theta}{r^{2}} \frac{\partial V(r, \theta)}{\partial \theta}=0$

For solving Equation (8) by separation of variable technique,

Let, $V(r, \theta)=R(r) G(\theta)$

Using equation (9) in equation (8) we get,

$\frac{\partial^{2} R(r) G(\theta)}{\partial r^{2}}+\frac{2}{r} \frac{\partial R(r)}{\partial r} G(\theta)+\frac{1}{r^{2}} R(r) \frac{\partial^{2} G(\theta)}{\partial \theta^{2}}+\frac{\cot \theta}{r^{2}} R(r) \frac{\partial G(\theta)}{\partial \theta}=0$

Dividing equation (10) by $\frac{R(r) G(\theta)}{r^{2}}$ and replacing $\partial / \partial r \rightarrow d / d r \& \partial / \partial \theta \rightarrow d / d \theta$ we get,

$\frac{1}{R(r)}\left[r^{2} \frac{d^{2}}{d r^{2}} R(r)+2 r \frac{d}{d r} R(r)\right]+\frac{1}{G(\theta)}\left[\frac{d^{2}}{d \theta^{2}} G(\theta)+\cot \theta \frac{d}{d \theta} G(\theta)\right]=0$

$\frac{1}{R(r)} \frac{d}{d r}\left[r^{2} \frac{d R(r)}{d r}\right]=-\frac{1}{G(\theta) \sin \theta} \frac{d}{d \theta}\left[\sin \theta \frac{d}{d \theta} G(\theta)\right]$

The left-hand side of equation (11) is the function of " $r$ " and the right-hand side is the function of " $\theta$ ". Since both sides of the equation (11) are equal, both sides should have a common constant value.

Let the constant value is " $\mathrm{K}$ ", So,

$$
\frac{1}{R(r)} \frac{d}{d r}\left(r^{2} \frac{d R(r)}{d r}\right)=K
$$

Or $\quad \frac{d}{d r}\left(r^{2} \frac{d R(r)}{d r}\right)-K R(r)=0$

and $\frac{1}{\sin \theta} \frac{d}{d \theta}\left[\sin \theta \frac{d G(\theta)}{d \theta}\right]+K G(\theta)=0$

These equations are known as Legendre's equation. The solution of equation (13) for all values of $\theta$ from 0 to $\pi$ is acceptable if $K=n(n+1)$, where $n$ is a positive integer. The solution of Legendre's equation is known as Legendre's polynomial, written as $P_{n}(\cos \theta)$.

Legendre's ploynomial can also be extended in orthogonal form as

$\int_{-1}^{1} P_{n}(x) P_{m}(x) d x=\frac{\delta_{m n}}{2 n+1} \quad$ where $x=\cos \theta$ 
$\delta_{m n}$ is Kronecker delta function.

$\delta_{m n}=\left\{\begin{array}{l}0 \text { for } n \neq m \\ 1 \text { for } n=m\end{array}\right.$

Therefore,

$P_{0}(\cos \theta)=1$,

$P_{1}(\cos \theta)=\cos \theta$,

$P_{2}(\cos \theta)=1 / 2\left(3 \cos ^{2} \theta-1\right)$,

$P_{3} \cos \theta=\frac{1}{2}\left(5 \cos ^{3} \theta-3 \cos \theta\right)$

The solution to the equation (12) is given by

$R(r)=A r^{n}+B r^{-(n+1)}$

The applied electric field across the cell gets distorted near the cell due to the cell's polarization. However, as the cell's distance increases, the distortion in the applied electric field decreases, as shown in Figure 4.

The electric field remains unchanged at a large distance from the cell. Therefore, $V(r)$ is directly proportional to the distance $\mathrm{r}$ from the cell.

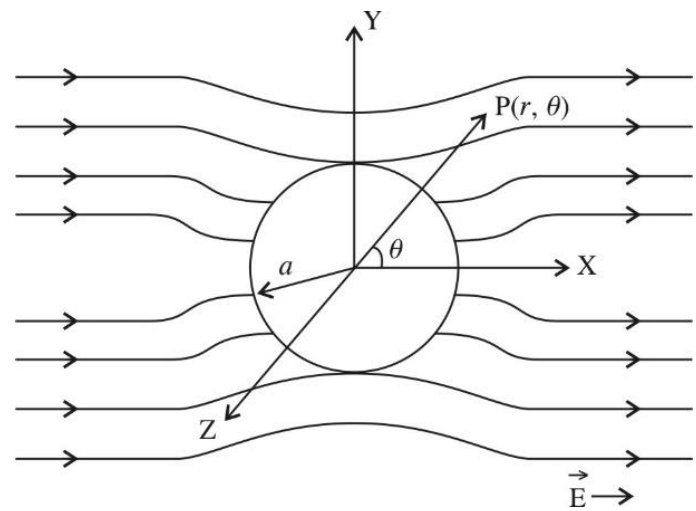

Figure 4. Distribution of electric field lines around a cell.

Therefore, equation (14) for $\mathrm{n}=1$ can be written as

$\mathrm{R}(\mathrm{r})=A r+\frac{B}{r^{2}}$

and $G(\theta)=P_{1}(\cos \theta)=\cos \theta$

So, equation (9) can be written as

$V(r, \theta)=\left(A r+\frac{B}{r^{2}}\right) \cos \theta$

The above equation is the general solution of $V(r, \theta)$.

The system under consideration consists of three regions: cytoplasm (c), cell membrane (m), and extracellular medium (e). Each region has a different electric potential; as such, the value of constants $\mathrm{A}$ and $\mathrm{B}$ in equation (15) are different for three different regions.

The above equations can also be written as:

$V(r, \theta)=\left\{\begin{array}{l}V_{c}(r, \theta)=\left(A_{c} r+\frac{B_{c}}{r^{2}}\right) \cos \theta, 0 \leq r \leq(a-h) \\ V_{m}(r, \theta)=\left(A_{m} r+\frac{B_{m}}{r^{2}}\right) \cos \theta,(a-h) \leq r \leq a \\ V_{e}(r, \theta)=\left(A_{e} r+\frac{B_{e}}{r^{2}}\right) \cos \theta, \quad r \geq a\end{array}\right.$

where "a" is the radius of the cell and " $\mathrm{h}$ " is the thickness of the cell membrane.

To further simplify the calculation, we need to apply boundary conditions. The boundary conditions are:

I. The voltage "V" is finite at $\mathrm{r}=0$, so $B_{c}=0$. 
II. The electric field is uniform at a very large distance " $r$ " from the cell's center.

Hence, $V(r, \theta)=-E r \cos \theta$

So, $A_{e}=-E$

Hence, equation (15) can be rewritten as

$$
V(r, \theta)=\left\{\begin{array}{lr}
V_{c}(r, \theta)=A_{c} r \cos \theta, & 0 \leq r \leq(a-h) \\
V_{m}(r, \theta)=\left(A_{m} r+\frac{B_{m}}{r^{2}}\right) \cos \theta, & (a-h) \leq r \leq a \\
V_{e}(r, \theta)=\left(-E r+\frac{B_{e}}{r^{2}}\right) \cos \theta, & r \geq a
\end{array}\right.
$$

III. The electric field and current densities are continuous at two interfaces between three regions.

a) $\left.V_{C}(r, \theta)\right|_{r=(a-h)}=\left.V_{m}(r, \theta)\right|_{r=(a-h)}$

So, equation (16) can be written as:

$$
A_{c}=A_{m}+\frac{B_{m}}{(a-h)^{3}}
$$

b) $\left.V_{c}(r, \theta)\right|_{r=a}=\left.V_{m}(r, \theta)\right|_{r=a}$

Therefore, $A_{m} a+\frac{B_{m}}{a^{2}}=-E_{a}+\frac{B_{e}}{a^{2}}$

Or $B_{e}=A_{m} a^{3}+B_{m}+E a^{3}$

c) $\left.\sigma_{c} \frac{\partial V(r, \theta)}{\partial r}\right|_{r=(a-h)}=\left.\sigma_{m} \frac{\partial V_{m}(r, \theta)}{\partial r}\right|_{r=(a-h)}$

Therefore, $A_{c}=\frac{\sigma_{m}}{\sigma_{c}} A_{m}-2\left(\frac{\sigma_{m}}{\sigma_{c}}\right) \frac{B_{m}}{(a-h)^{3}}$

d) $\left.\sigma_{m} \frac{\partial V_{m}(r, \theta)}{\partial r}\right|_{r=a}=\left.\sigma_{e} \frac{\partial V_{c}}{\partial r}\right|_{r=a}$

Therefore, $\frac{\sigma_{m}}{\sigma_{c}}\left[A_{m}-\frac{2 B_{m}}{a^{3}}\right]+E=-\frac{2 B_{c}}{a^{3}}$

Using equation (18), we get,

$$
A_{m}\left(\sigma_{m}+2 \sigma_{e}\right)=\frac{2 B_{m}}{a^{3}}\left(\sigma_{m}-\sigma_{e}\right)-3 E \sigma_{e}
$$

From the equation (17) and (19), we get,

or $\quad A_{m}\left(\sigma_{c}-\sigma_{m}\right)=\frac{-B_{m}\left(2 \sigma_{m}+\sigma_{c}\right)}{(a-h)^{3}}$

$$
A_{m}+\frac{B_{m}}{(a-h)^{3}}=\frac{\sigma_{m}}{\sigma_{c}} A_{m}-2\left(\frac{\sigma_{m}}{\sigma_{c}}\right) \frac{B_{m}}{(a-h)^{3}}
$$

Dividing equations (21) and (22), we get

$$
B_{m}=\frac{3 a^{3}(a-h)^{3} E \sigma_{e}\left(\sigma_{c}-\sigma_{m}\right)}{2 a^{3}\left(\sigma_{m}+2 \sigma_{e}\right)\left(\sigma_{m}+\frac{\sigma_{c}}{2}\right)-2(a-h)^{3}\left(\sigma_{e}-\sigma_{m}\right)\left(\sigma_{c}-\sigma_{m}\right)}
$$

Using equation (23) in (21), we get

$$
A_{m}=\frac{-3 E a^{3} \sigma_{e}\left(\sigma_{c}+2 \sigma_{m}\right)}{2 a^{3}\left(\sigma_{m}+2 \sigma_{e}\right)\left(\sigma_{m}+\frac{\sigma_{c}}{2}\right)-2(a-h)^{3}\left(\sigma_{e}-\sigma_{m}\right)\left(\sigma_{c}-\sigma_{m}\right)}
$$

Using equations (23) and (24) in equation (17), we get

$$
A_{c}=\frac{-9 E a^{3} \sigma_{e} \sigma_{m}}{2 a^{3}\left(\sigma_{m}+2 \sigma_{e}\right)\left(\sigma_{m}+\frac{\sigma_{c}}{2}\right)-2(a-h)^{3}\left(\sigma_{e}-\sigma_{m}\right)\left(\sigma_{c}-\sigma_{m}\right)}
$$

Using equations (23) and (24) in equation (18), we get

$$
B_{e}=\frac{E a^{3}\left[a^{3}\left(\sigma_{m}-\sigma_{e}\right)\left(2 \sigma_{m}+\sigma_{c}\right)-(a-h)^{3}\left(\sigma_{m}-\sigma_{c}\right)\left(2 \sigma_{m}+\sigma_{e}\right)\right]}{2 a^{3}\left(\sigma_{m}+2 \sigma_{e}\right)\left(\sigma_{m}+\frac{\sigma_{c}}{2}\right)-2(a-h)^{3}\left(\sigma_{e}-\sigma_{m}\right)\left(\sigma_{c}-\sigma_{m}\right)}
$$

Using the values of constants $A_{m}$ and $B_{m}$ in equation (16), we get

$$
V_{m}(a-h, \theta)=\left(A_{m}(a-h)+\frac{B_{m}}{(a-h)^{2}}\right) \cos \theta
$$

On solving the equation by using values from equations (23) and (24), we get 
$V_{m}(a-h, \theta)=\left[\frac{-9 E a^{3}(a-h)^{3} \sigma_{e} \sigma_{m}}{2(a-h)^{2} a^{3}\left(\sigma_{m}+2 \sigma_{e}\right)\left(\sigma_{m}+\frac{\sigma_{c}}{2}\right)-2(a-h)^{3}\left(\sigma_{e}-\sigma_{m}\right)\left(\sigma_{c}-\sigma_{m}\right)}\right] \cos \theta$

and $\quad V_{m}(a, \theta)=\frac{-3 E a^{3} \sigma_{e}\left[a^{3}\left(\sigma_{c}+2 \sigma_{m}\right)-(a-h)^{3}\left(\sigma_{c}-\sigma_{m}\right)\right]}{a^{2}\left[2 a^{3}\left(\sigma_{m}+2 \sigma_{e}\right)\left(\sigma_{m}+\frac{\sigma_{c}}{2}\right)-2(a-h)^{3}\left(\sigma_{e}-\sigma_{m}\right)\left(\sigma_{c}-\sigma_{m}\right)\right]} \cos \theta$

The induced transmembrane voltage (ITMV) is given by

$\Delta V_{m}=V_{m}(a-h, \theta)-V_{m}(a, \theta)$

On solving the above equation and by reducing it we get,

$\Delta V_{m}=\frac{3 \sigma_{e}\left[3 a^{2} h \sigma_{c}+h^{2}(3 a-h)\left(\sigma_{m}-\sigma_{c}\right)\right] E a \cos \theta}{2 a^{3}\left(\sigma_{m}+2 \sigma_{e}\right)\left(\sigma_{m}+\frac{\sigma_{c}}{2}\right)-2(a-h)^{3}\left(\sigma_{e}-\sigma_{m}\right)\left(\sigma_{c}-\sigma_{m}\right)}$

This is also written as

$\Delta V_{m}=f_{s} E a \cos \theta$

where, $f_{s}=\frac{3 \sigma_{e}\left[3 a^{2} h \sigma_{c}+h^{2}(3 a-h)\left(\sigma_{m}-\sigma_{c}\right)\right]}{2 a^{3}\left(\sigma_{m}+2 \sigma_{e}\right)\left(\sigma_{m}+\frac{\sigma_{c}}{2}\right)-2(a-h)^{3}\left(\sigma_{e}-\sigma_{m}\right)\left(\sigma_{c}-\sigma_{m}\right)}$ $f_{S}=\frac{3}{2}$

Since plasma membrane is dielectric $\left(\sigma_{m}=0\right)$, therefore, equation (29) is reduced to

So equation (28) can be written as

$\Delta V_{m}=\frac{3}{2} E a \cos \theta$

This equation is the steady-state Schwan's equation.

Thus, ITMV depends upon the cell radius, the magnitude of the applied electric field, and the orientation of the cell.

The positions of the spherical cell corresponding to $\theta$ to $0^{0}$ and $180^{\circ}$ with respect to the electric field are called poles of the cell, and values of ITMV at poles are given by.

$$
\Delta V_{m}=\begin{gathered}
+\frac{3}{2} \text { Ea for } \theta=0^{0} \\
-\frac{3}{2} \text { Ea for } \theta=180^{\circ}
\end{gathered}
$$

Thus, TMV values are maximum at poles of the spherical cell subjected to the DC electric field for hundreds of $\mu$ s. The variation of $\Delta V_{m}$ with angle $\theta$ for a spherical cell and constant electric field "E" is shown in Figure 4.

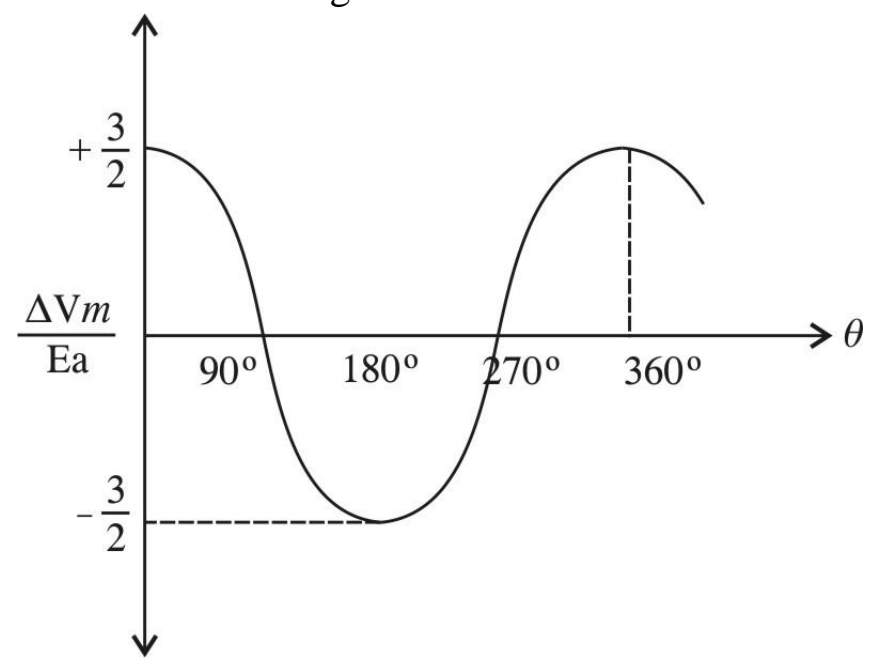

Figure 5. The variation of $\Delta V_{m}$ with angle $\theta$ for a spherical cell.

The transient behavior of the cell in the initial microseconds can be described by taking into consideration the dielectric permittivity of the membrane $\varepsilon_{m}$ in addition to the electric conductivity. 
Hence, the equation of ITMV can be modified as

$$
\Delta V_{m}=\frac{3}{2} E a \cos \theta\left(1-e^{\frac{-t}{\tau_{m}}}\right)
$$

where $\tau_{m}$ is the membrane charging time and can be expressed as

$$
\tau_{m}=R_{c} C_{m}=\frac{a \varepsilon_{m}}{2 h\left(\frac{\sigma_{c} \sigma_{e}}{\sigma_{c}+2 \sigma_{e}}\right)+a \sigma_{m}}
$$

The membrane charging time is the time between the applied electric field and the induced TMV. The time delay under normal physiological conditions is less than $\mu$ s but can be increased in the case of cells suspended in a low conductive medium. For AC electric field having a time period greater than $\tau_{m}$ and for rectangular pulses longer than $\tau_{m}$, the amplitude of ITMV is given by

$$
\Delta V_{m}=\frac{3}{2} E a \cos \theta
$$

For AC electric field having time period equal to or comparable to $\tau_{m}$ is given by,

$$
\Delta V_{m}=\frac{3}{2} E a \cos \theta\left(1-\frac{1}{e}\right)
$$

Thus, we can conclude that the ITMV gets decreased exponentially with the application of the pulsed electric field.

\subsection{COMSOL simulation of molecular transport.}

To further investigate the molecular transport in an electroporated Schwan cell, we simulated the cell using the parameters mentioned in Table 1 in COMSOL Multiphysics 5.3.

To calculate the value of the electric field that is needed to be applied, we first set the ITMV $V_{m}$ to $1 \mathrm{~V}$ and $\theta$ to be 00 . The applied electric field is calculated to be $6.17 \mathrm{~V}$. The calculations were made by considering the $30 \mu \mathrm{m}$ electrode gap [26]. The result of the simulation is shown in Figure 6.

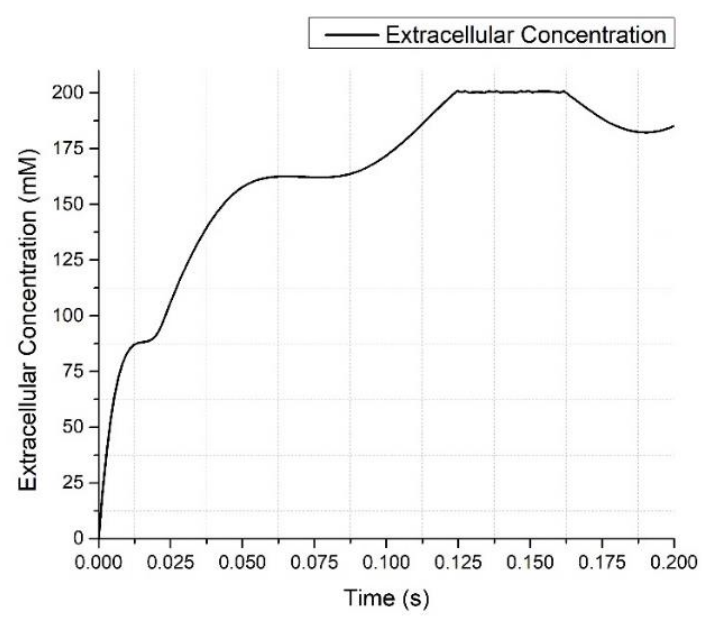

Figure 6. Time vs. intracellular concentration.

From the graph, it is evident that the concentration of molecules started to increase with the electric field application. It has reached a maximum concentration of $200 \mathrm{mM}$. The concentration started to drop to $175 \mathrm{mM}$ as the applied electric field started to decrease.

Thus, we can conclude that electroporation results in the transport of molecules into the intracellular space and can be used for DNA vaccination and plasmid delivery once the ITMV is calculated and a closed-form expression is derived. 


\section{Conclusions}

This paper derives the close form expression for induced transmembrane voltage. The derivation includes the utilization of Gauss's law for the calculation of electric field distribution. The solution is obtained by converting the equation to Laplace's equation and solving it to calculate ITMV. The close loop expression is further used to evaluate the molecular transport mechanism in Multiphysics software.

The results obtained from the simulation significantly match with the extracellular concentration profile obtained by the empirical methods from the literature. The close form expression evolved in this paper helps in defining parameters for electroporation-assisted vaccine delivery of Covid-19. The simulation presents a time-efficient method for vaccine development in comparison to experimental methods.

\section{Funding}

This research received no external funding.

\section{Acknowledgments}

The authors would like to acknowledge the Chitkara University Research \& Innovation Network (CURIN) for offering computational facilities in their laboratory.

\section{Conflicts of Interest}

The authors declare no conflict of interest.

\section{References}

1. Kotnik, T.; Rems, L.; Tarek, M.; Miklavčič, D. Membrane Electroporation and Electropermeabilization: Mechanisms and Models. Annu. Rev. Biophys. 2019, 48, 63-91, https://doi.org/10.1146/annurev-biophys052118-115451.

2. Li, C.; Ke, Q.; Yao, C.; Mi, Y.; Liu, H.; Lv, Y.; Yao, C. Cell Electrofusion Based on Nanosecond/Microsecond Pulsed Electric Fields. PLoS One 2018, 13, e0197167, https://doi.org/10.1371/journal.pone.0197167.

3. Stewart, M.P.; Langer, R.; Jensen, K.F. Intracellular Delivery by Membrane Disruption: Mechanisms, Strategies, and Concepts. Chem. Rev. 2018, 118, 7409-7531, https://doi.org/10.1021/acs.chemrev.7b00678.

4. Kašèta, V.; Kaušylè, A.; Kavaliauskaitè, J.; Petreikytè, M.; Stirkè, A.; Biziulevičienè, G. Detection of Intracellular Biomarkers in Viable Cells Using Millisecond Pulsed Electric Fields. Exp. Cell Res. 2020, 389, 111877, https://doi.org/10.1016/j.yexcr.2020.111877.

5. Whalley, N.A.; Walters, S.; Hammond, K. Molecular Cell Biology. In Molecular Medicine for Clinicians; 2018.

6. Alberts, B.; Johnson, A.; Lewis, J.; Morgan, D.; Raff, M.; Roberts, K.; Walter, P. Membrane Transport of Small Molecules and the Electrical Properties of Membranes. In Molecular Biology of the Cell; Wilson, J., Hunt, T., Eds.; Garland Science, 2017; 597-640.

7. Clark, D.P.; Pazdernik, N.J.; McGehee, M.R. Mutations and Repair. Molecular Biology, Elsevier, Amsterdam 2019, 832-879.

8. Vižintin, A.; Marković, S.; Ščančar, J.; Miklavčič, D. Electroporation with nanosecond pulses and bleomycin or cisplatin results in efficient cell kill and low metal release from electrodes. Bioelectrochemistry 2021, 140, 107798, doi:10.1016/j.bioelechem.2021.107798.

9. Neumann, E.; Schaefer-Ridder, M.; Wang, Y.; Hofschneider, P.H. Gene Transfer into Mouse Lyoma Cells by Electroporation in High Electric Fields. EMBO J. 1982, 1, 841-845, https://doi.org/10.1002/j.14602075.1982.tb01257.x.

10. Romeo, S.; Sannino, A.; Scarfì, M.R.; Vernier, P.T.; Cadossi, R.; Gehl, J.; Zeni, O. ESOPE-Equivalent 
Pulsing Protocols for Calcium Electroporation: An In Vitro Optimization Study on 2 Cancer Cell Models. Technol. Cancer Res. Treat. 2018, 17, 153303381878807, https://doi.org/10.1177/1533033818788072.

11. Gehl, J.; Sersa, G.; Matthiessen, L.W.; Muir, T.; Soden, D.; Occhini, A.; Quaglino, P.; Curatolo, P.; Campana, L.G.; Kunte, C.; Clover, A.J.P.; Bertino, G.; Farricha, V.; Odili, J.; Dahlstrom, K.; Benazzo, M.; Mir, L.M. Updated standard operating procedures for electrochemotherapy of cutaneous tumours and skin metastases. Acta Oncol. 2018, 57, 874-882, https://doi.org/10.1080/0284186X.2018.1454602.

12. Kiełbik, A.; Szlasa, W.; Michel, O.; Szewczyk, A.; Tarek, M.; Saczko, J.; Kulbacka, J. In Vitro Study of Calcium Microsecond Electroporation of Prostate Adenocarcinoma Cells. Molecules 2020, 25, 5406, https://doi.org/10.3390/molecules25225406.

13. Ghosh, D.; Gurjeet Singh, T.; Saluja, N. A Critical Analysis Of Electroporation In Medical Technology Numerical Analysis Of Electroporation View Project A Critical Analysis Of Electroporation In Medical Technology. Artic. Int. J. Pharm. Sci. Res. 2019, 10, 23, https://doi.org/10.13040/IJPSR.0975-8232.10(1).2328.

14. Nemec, A.; Milevoj, N.; Lampreht Tratar, U.; Serša, G.; Čemažar, M.; Tozon, N. Electroporation-Based Treatments in Small Animal Veterinary Oral and Maxillofacial Oncology. Front. Vet. Sci. 2020, 7 , https://doi.org/10.3389/fvets.2020.575911.

15. Marino, M.; Luján, E.; Mocskos, E.; Marshall, G. OpenEP: An Open-Source Simulator for ElectroporationBased Tumor Treatments. Sci. Rep. 2021, 11, 1423, https://doi.org/10.1038/s41598-020-79858-y.

16. Geboers, B.; Scheffer, H.J.; Graybill, P.M.; Ruarus, A.H.; Nieuwenhuizen, S.; Puijk, R.S.; van den Tol, P.M.; Davalos, R.V.; Rubinsky, B.; de Gruijl, T.D.; Miklavčič, D.; Meijerink, M.R. High-Voltage Electrical Pulses in Oncology: Irreversible Electroporation, Electrochemotherapy, Gene Electrotransfer, Electrofusion, and Electroimmunotherapy. Radiology 2020, 295, 254-272, https://doi.org/10.1148/radiol.2020192190.

17. Novickij, V.; Rembialkowska, N.; Staigvila, G.; Kulbacka, J. Effects of Extracellular Medium Conductivity on Cell Response in the Context of Sub-Microsecond Range Calcium Electroporation. Sci. Rep. 2020, 10, 3718, https://doi.org/10.1038/s41598-020-60789-7.

18. Navickaite, D.; Ruzgys, P.; Novickij, V.; Jakutaviciute, M.; Maciulevicius, M.; Sinceviciute, R.; Satkauskas, S. Extracellular-Ca2+-Induced Decrease in Small Molecule Electrotransfer Efficiency: Comparison between Microsecond and Nanosecond Electric Pulses. Pharmaceutics 2020, 12, 422, https://doi.org/10.3390/pharmaceutics12050422.

19. Miklavčič, D. Handbook of Electroporation; Miklavčič, D., Ed.; Springer International Publishing: Cham, 2017; ISBN 978-3-319-32885-0.

20. Tebas, P.; Yang, S.; Boyer, J.D.; Reuschel, E.L.; Patel, A.; Christensen-Quick, A.; Andrade, V.M.; Morrow, M.P.; Kraynyak, K.; Agnes, J.; Purwar, M.; Sylvester, A.; Pawlicki, J.; Gillespie, E.; Maricic, I.; Zaidi, F.I.; Kim, K.Y.; Dia, Y.; Frase, D.; Pezzoli, P.; Schultheis, K.; Smith, T.R.F.; Ramos, S.J.; McMullan, T.; Buttigieg, K.; Carroll, M.W.; Ervin, J.; Diehl, M.C.; Blackwood, E.; Mammen, M.P.; Lee, J.; Dallas, M.J.; Brown, A.S.; Shea, J.E.; Kim, J.J.; Weiner, D.B.; Broderick, K.E.; Humeau, L.M. Safety and immunogenicity of INO-4800 DNA vaccine against SARS-CoV-2: A preliminary report of an open-label, Phase 1 clinical trial. EClinicalMedicine 2021, 31, 100689, https://doi.org/10.1016/j.eclinm.2020.100689.

21. Pavlin, M.; Kotnik, T.; Miklavčič, D.; Kramar, P.; Maček Lebar, A. Chapter Seven Electroporation of Planar Lipid Bilayers and Membranes. In Advances in Planar Lipid Bilayers and Liposomes; 2008; 165-226.

22. Zou, Y.; Wang, C.; Peng, R.; Wang, L.; Hu, X. Theoretical Analyses of Cellular Transmembrane Voltage in Suspensions Induced by High-Frequency Fields. Bioelectrochemistry 2015, https://doi.org/10.1016/j.bioelechem.2014.12.002.

23. Guo, F.; Zhang, L.; Liu, X. Nonlinear Dispersive Cell Model for Microdosimetry of Nanosecond Pulsed Electric Fields. Sci. Rep. 2020, 10, 19456, https://doi.org/10.1038/s41598-020-76642-w.

24. Shi, F.; Kolb, J.F. Enhanced Resolution Impedimetric Analysis of Cell Responses from the Distribution of Relaxation Times. Biosens. Bioelectron. 2020, 157, 112149, https://doi.org/10.1016/j.bios.2020.112149.

25. Schwan, H.P. Electrical Properties of Tissue and Cell Suspensions. In Advances in biological and medical physics; Elsevier, 1957; 5, 147-209.

26. Jayasooriya, V.; Nawarathna, D. Simulation of Molecular Transport through an Electroporated Cell Using COMSOL Multiphysics. COMSOL Conf. 2017. 